\title{
Transanal endorectal stepwise gradient muscular cuff cutting pull-through method: Technique refinements and comparison with laparoscopy-assisted procedures
}

\author{
ZEBING ZHENG ${ }^{1}$, FAN ZHANG ${ }^{2}$, ZHU JIN $^{1}$, MINGJUAN GAO ${ }^{1}$, YUCHEN MAO ${ }^{1}$, YAN QU ${ }^{1}$ and YUANMEI LIU ${ }^{1}$ \\ Departments of ${ }^{1}$ Pediatric General Thoracic and Urinary Surgery and ${ }^{2}$ Anesthesiology, \\ Affiliated Hospital of Zunyi Medical College, Zunyi, Guizhou 563000, P.R. China
}

Received August 20, 2017; Accepted June 8, 2018

DOI: $10.3892 /$ etm.2018.6414

\begin{abstract}
In all existing radical resection procedures available for Hirschprung's disease (HD), the muscular cuff has been retained. In recent years, our study group has modified the procedure using a stepwise gradient muscular cuff cutting pull-through method for the treatment of HD. The objective of the present study was to assess patient prognosis following the use of the transanal endorectal pull-through (TEPT) method or the laparoscopy-assisted pull-through (LPT) method and to provide evidence to assist in clinical decisions. The medical records of 172 patients (age, $\geq 3$ years) who underwent TEPT or LPT for HD between September 2003 and August 2014 at the Pediatric Surgery Department of The Affiliated Hospital of Zunyi Medical College (Zunyi, China) were reviewed (TEPT, n=94; LPT, n=78). Preoperative, intraoperative and post-operative data for the subjects involved were recorded. Each patient's family was interviewed using a uniform 12-item post-pull-through long-term outcome questionnaire, which was scored in order to compare the short and long-term treatment outcomes of patients in each group. Patients in the LPT group had significantly prolonged operating times, reduced intraoperative blood loss and perioperative transfusion rates and shorter anal dissection times. Furthermore, the oral feeding time and length of hospital stay were similar between groups. There were no significant differences in early complications between the groups. No significant differences in late complications, including anastomotic stricture and enterocolitis were observed; however, the soiling and constipation rates were significantly higher in the TEPT group compared with the LPT group. The stool pattern score was not significantly
\end{abstract}

Correspondence to: Dr Yuanmei Liu, Department of Pediatric General Thoracic and Urinary Surgery, Affiliated Hospital of Zunyi Medical College, 149 Dalian Road, Zunyi, Guizhou 563000, P.R. China

E-mail: yuanmei116@aliyun.com

Key words: Hirschsprung's disease, transanal, laparoscopic, rectal muscular cuff, long-term outcome different between groups, while the frequency of bowel movements in the LPT group was significantly higher compared with the TEPT group from 9-24 months post-surgery. The stepwise gradient muscular cuff cutting pull-through method demonstrated a good safety profile and efficacy with regard to the long-term outcomes of patients with HD. These results support the clinical application of LPT as a promising surgical approach for children with HD.

\section{Introduction}

Hirschsprung's disease (HD) is characterized by the absence of ganglion cells in the distal bowel. In recent years, various operative modes have been developed for the surgical treatment of HD (1-3). The incidence of HD is $~ 1 / 5,000$ live births and men are 3.5-7.8 times more likely to be affected than women. HD usually occurs in infancy, although some people will present with persistent, severe constipation in the later life (4). This condition is confined to the rectosigmoid colon in $75 \%$ of affected patients. The rectosigmoid colon variant is the most frequent presentation, but an extended form has also been observed. In $17 \%$ of all cases, the aganglionic segment is observed to extend from the rectum, sigmoid colon and colon up to part of the transverse colon. The rarest form of the disease with the most severe clinical course is total colonic aganglionosis (TCA), which is observed in $8 \%$ of patients (5).

Surgical intervention for HD involves the removal of the aganglionic bowel to provide normally innervated intestine to the anus (6). Classical pull-through techniques as described by Soave et al have been modified and improved in order to reduce the surgical incision range, minimize damage to the surrounding structures during intra-abdominal dissection and eliminate associated complications $(5,7)$. The transanal endorectal pull-through (TEPT) technique was subsequently proposed by De la Torre-Mondragon and Ortega-Salgado, which revolutionized HD treatment (8). In 1995, the one stage laparoscopy-assisted pull-through (LPT) approach was proposed by Georgeson et al (9) and has since been extensively applied for the treatment of HD. This method has several merits, including no need for colostomy, and a lack of intraperitoneal adherence and/or scarring (10). While LPT can be performed for patients with different types of HD, it is more 
conventionally applied to treat aganglionosis, which extends to the ascending colon (11). At present, there are several surgical techniques available for the treatment of HD; however, their outcomes remain unsatisfactory $(12,13)$.

Between September 2003 and August 2014, all patients with HD at the Pediatric Surgery Department of The Affiliated Hospital of Zunyi Medical College (Zunyi, China) were treated using the stepwise and gradient muscular cuff cutting method. TEPT or LPT surgical techniques were selected based on the preference of the patients' families, as well as the experience of the surgeon. In the present study, a novel type of pull-through operative method is presented. A novel technique for refinement is also proposed and the short- and long-term impacts of the two methods are compared with the aim of developing a novel and effective treatment technique for pediatric surgeons.

\section{Materials and methods}

Patients. The study protocol was formally approved by the Ethics Committee of The Affiliated Hospital of Zunyi Medical College. All patients' families gave their informed consent. The medical records of 221 children admitted to the institution between September 2003 and August 2014 with a diagnosis of HD were retrospectively reviewed. All diagnoses were identified based on the histological examination of rectal biopsy samples. A number of patients were excluded, including those who had received conventional transabdominal pull-through procedures $(n=24)$, patients who were diagnosed with TCA $(n=7)$ and those who followed up abroad halfway $(n=18)$. Consequently, 172 patients (mean age, 13.5 months; male:female, 138:34) were examined, all of whom were eventually treated with the stepwise and gradient muscular cuff cutting method for HD. Ultimately, 78 and 94 children were treated using the LPT and TEPT techniques, respectively. The data collected includes the baseline index, clinical presentation, type of surgery performed, systems review (i.e., mortality and quality of life), operative details, time to oral feeding, duration of hospitalization, length of intensive care unit (ICU) stay, early and late postoperative complications, episodes of postoperative enterocolitis and stooling habits (i.e., continence, frequency, requirement of laxatives or other medications). The enterocolitis severity was graded using a previously designed scoring system from mild (i.e., grade I) to severe (i.e., grade III) (14). Follow-up information was obtained from outpatient visit notes, as well as telephone questionnaire feedback. Patient/family surveys were conducted for patients older than 3 years and scored using an improved uniform 12-item long-term prognosis survey following the pull-through procedure (15) (Table I).

Surgical techniques (TEPT group). A full TEPT method was used. Patients were examined with serial rectal lavage and anal dilation was performed via digital dilatations prior to surgery. The final rectal washouts contained metronidazole $(25 \mathrm{mg} / \mathrm{kg}$ body weight; Shijiazhuang No. 4 Pharmaceutical Co., Ltd., Hebei, China). Third-generation cephalosporin antibiotics (Cefotaxime Sodium for Injection; $50 \mathrm{mg} / \mathrm{kg}$ body weight; NCPC Hebei Huamin Pharmaceutical Co., Ltd., Hebei, China) were administered $30 \mathrm{~min}$ prior to surgery. Patients were placed in a supine position with dangling legs throughout the operation. Anal retractors were used around the anal verge to expose the anus. A 4-0 polypropylene monofilament prolene suture was placed through the tip of the glans as a traction suture within $0.5-1.0 \mathrm{~cm}$ above the dentate line. Fascicular electrocautery was performed for the delicate dissection of rectal muscle and hemostasis of the main vessels in an effort to avoid damage to the pelvic nerves. In the present study, a novel technique was refined; this involved cutting the rectal circular muscle within $1-2 \mathrm{~cm}$ of the starting point of the submucosal dissection. First, the rectal longitudinal smooth muscle was cut and the rectum ring muscle was separated after an incision of 1-2 cm. The anal sphincter was also protected. This differs from traditional Soave operations in which the whole muscle cuff remains and the anal sphincter is usually damaged (16). The surgery was modified by the authors of the current study since the longitudinal rectal muscle was cut $2-3 \mathrm{~cm}$ above the circular muscle. Using a surgical incision of the stepwise gradient muscular cuff up towards the peritoneal cavity, the muscular cuff remained $1-2 \mathrm{~cm}$ in neonates and infants, or $3-4 \mathrm{~cm}$ in children. The aganglionic bowel was pulled through the muscular cuff and the bowel was separated to the normal zone. Once the biopsy site demonstrating normal innervation was reached, anastomosis was performed outside the anal cavity. The muscular cuff was then sutured with the normal bowel to prevent mucosa prolapsing. The colonal anastomosis with the mucosa of the dentate line exhibited a heart-shaped anastomosis, higher in the front and lower in the back. At 2 weeks post-surgery, gentle rectal dilatations were performed using a dilator.

LPT group. The LPT procedure was performed for patients with HD confined to the left colon based on a preoperative barium enema examination. All patients who received the LPT procedure were in a similar condition prior to surgery as the patients in the TEPT group. Three trocars were used and a transumbilical camera port (e.g., $5 \mathrm{~mm}$ ) and two operative ports ( $3 \mathrm{~mm}$ or $5 \mathrm{~mm}$ ) were inserted bilaterally two-thirds into the line from the umbilicus to the anterior superior iliac spine. In the first step, multiple seromuscular leveling biopsies of the bowel were obtained in order to pathologically detect ganglion cells in the submucosal nerve plexus. The length of abscission was also determined based on biopsies that confirmed the absence of ganglion cells in sections of the colon; typical histological findings for HD in a rectal biopsy include the absence of ganglion cells in the submucosal (Meissner's) plexus and the intermuscular (Auerbach's) plexus $(17,18)$. The mesentery was separated from the rectal wall to protect the lower rectal vessels and maintain a good blood supply to the rectal muscular sheath. Dissection was followed to the peritoneal reflection of the rectum. Endorectal dissection was the same as performed in the TEPT group. In the present study, mucosectomy was frequently performed prior to the development of the stepwise and gradient cutting muscular cuff dissected rectum method.

Post-surgical care. Following, surgery, all patients received intravenous broad-spectrum antibiotics and an injection of metronidazole $(25 \mathrm{mg} / \mathrm{kg})$ to prevent enterocolitis. In the majority of patients, an anal supporting tube was inserted and maintained for 5-7 days to prevent anastomotic stricture and enterocolitis. At 2 weeks post-surgery, patients received 
Table I. Telephone questionnaire scores for patients older than 3 years.

\begin{tabular}{|c|c|c|}
\hline Question & Answer & Score \\
\hline \multirow[t]{8}{*}{ Frequency of defecation } & Only with enema & 4 \\
\hline & Only with suppository & 3 \\
\hline & Every 3 day or more but spontaneous & 2 \\
\hline & Every 1-2 day & 1 \\
\hline & Normal (1-2/day) & 0 \\
\hline & Often (3-5/day) & 1 \\
\hline & $6-7 / d$ & 2 \\
\hline & 8 or more movements/day & 3 \\
\hline \multirow[t]{4}{*}{ Soling } & Permanent (more than 6 times/day) & 3 \\
\hline & Often (4-6 times/day) & 2 \\
\hline & Occasional (1-3 times/day) & 1 \\
\hline & None & 0 \\
\hline \multirow[t]{3}{*}{ Times of soling } & At night and day & 2 \\
\hline & At night or activity & 1 \\
\hline & None & 0 \\
\hline \multirow[t]{3}{*}{ Is he/she fully continent? } & Not continent & 2 \\
\hline & Partially (occasional accidents) & 1 \\
\hline & Yes & 0 \\
\hline \multirow[t]{4}{*}{ Stool consistency } & Liquid & 3 \\
\hline & Hard & 2 \\
\hline & Loose & 1 \\
\hline & Normal & 0 \\
\hline \multirow{2}{*}{ Stool odor } & Offensive odor & 1 \\
\hline & Normal odor & 0 \\
\hline \multirow[t]{4}{*}{ Loss of stool during coughing or crying } & Solid & 3 \\
\hline & Liquid & 2 \\
\hline & Gas & 1 \\
\hline & No & 0 \\
\hline \multirow[t]{3}{*}{ Requires diapers } & Continuously & 2 \\
\hline & Night or activity & 1 \\
\hline & None & 0 \\
\hline \multirow[t]{3}{*}{ Sense of fullness and evacuation after defecation } & Absent sense of fullness & 2 \\
\hline & Fullness but partial evacuation & 1 \\
\hline & Fullness and evacuation & 0 \\
\hline \multirow[t]{3}{*}{ Distension } & Moderate to severe & 2 \\
\hline & Mild & 1 \\
\hline & No & 0 \\
\hline \multirow[t]{4}{*}{ Need for medical therapy to control stooling } & Always & 3 \\
\hline & Occasionally & 2 \\
\hline & Long period but finally weaned off & 1 \\
\hline & No & 0 \\
\hline \multirow[t]{4}{*}{ Recurrent attacks of enterocolitis } & More than 6 attacks & 3 \\
\hline & 4-6 attacks & 2 \\
\hline & 1-3 attacks & 1 \\
\hline & None & 0 \\
\hline
\end{tabular}

a routine anal expanding program with a Hegar dilator for 2 months.
Statistical analysis. All data are expressed as the mean \pm standard deviation and statistical analysis was 
performed using SPSS software version 19.0 (IBM SPSS Software, Armonk, NY, USA). The Student's t-test was used to compare different parameters (i.e., age, weight, operative time, intraoperative blood loss, anal dissection time, oral feeding time and duration of hospitalization) between the LPT and TEPT groups. Sex, aganglionic segment, early post-operative complications, late post-operative complications and bowel movements were also compared using the chi-squared test. $\mathrm{P}<0.05$ was considered to indicate a statistically significant difference.

\section{Results}

Patient characteristics. In the present study, 172 patients over a 10-year period underwent surgery using a stepwise and gradient cutting muscular cuff method with a pull-through procedure. Their general clinical data are presented in Table II. No significant differences were observed in sex, age or weight. Aganglionic segments were discovered extending to the rectosigmoid, descending colon, transverse colon and ascending colon in the two groups.

Operative data. The operative data and post-operative outcomes are presented in Fig. 1. Patients in the TEPT group had significantly shorter operative times $(117.6 \pm 16.0 \mathrm{~min})$ compared with the LPT group $(135.5 \pm 35.0 \mathrm{~min} ; \mathrm{P}<0.05$; Fig. 1A). However, in the LPT group intraoperative blood loss $(15.8 \pm 10.0 \mathrm{ml}$ vs. $27.9 \pm 14.1 \mathrm{ml}$; $\mathrm{P}<0.05$; Fig. $1 \mathrm{~B})$ and anal dissection times $(44.4 \pm 7.5$ min vs. $71.4 \pm 9.6 \mathrm{~min} ; \mathrm{P}<0.05$; Fig. 1C) were significantly decreased compared with the TEPT group. No significant differences were observed in oral feeding time or duration of hospitalization (Fig. 1D and E).

Post-operative complications. The early post-operative complications are listed in Table III. A total of 27 patients $(25.6 \%)$ in the TEPT group and 20 patients $(28.7 \%)$ in the LPT group presented with perianal dermatitis in the early post-operative period. There was no significant difference in the incidence of perianal dermatitis between the two groups. There were 4 patients (4.3\%) in the TEPT group and 2 patients (2.6\%) in the LPT group who exhibited post-operative uroschesis; however, no significant difference was observed. No patients were diagnosed with anastomotic leakage or anastomotic structures. Additionally, 2 patients in the LPT group had recurrent intestinal obstruction caused by adhesion and 1 patient exhibited intestinal volvulus of the pull-through rectal area in the early post-operative period, which was treated using adhesiolysis and colonic anastomosis through laparotomy; none of these events were recorded in the TEPT group $(\mathrm{P}=0.055)$.

Enterocolitis occurred in both groups, with 19 (20.2\%) and $25(32.1 \%)$ cases in the TEPT and LPT groups, respectively $(\mathrm{P}=0.076)$. The severity of these episodes did not significantly differ between the two groups. Symptoms were improved following metronidazole administration via a coloclyster, gastrointestinal decompression, antibiotic treatment, parenteral nutritional support and regulation of intestinal microflora.

Follow-up. Patients were followed up post-operatively for 36-120 months. Long-term post-operative complications are
Table II. Clinical and demographic characteristics.

\begin{tabular}{lccc}
\hline Parameter & TEPT $(\mathrm{n}=94)$ & LPT $(\mathrm{n}=78)$ & P-value \\
\hline Age at surgery (months) & $15.4 \pm 15.7$ & $11.5 \pm 12.7$ & 0.081 \\
Sex (male:female) & $78: 16$ & $60: 18$ & 0.321 \\
Weight at surgery (kg) & $8.7 \pm 4.0$ & $7.6 \pm 3.6$ & 0.074 \\
Aganglionic segment & & & \\
Ultrashort & 21 & 10 & 0.106 \\
Rectosigmoid & 45 & 35 & 0.694 \\
Descending colon & 24 & 17 & 0.567 \\
Transverse colon & 4 & 12 & 0.017 \\
Ascending colon & 0 & 4 & 0.026
\end{tabular}

TEPT, transanal endorectal stepwise and gradient cutting muscular cuff pull-through; LPT, laparoscopy-assisted Soave pull-through.

listed in Table IV. A total of 8 patients in the TEPT group $(8.5 \%)$ and 7 patients in the LPT group (9.0\%) had recurrent anastomotic strictures in the late post-operative period $(\mathrm{P}=0.915)$. Furthermore, 2 patients in the TEPT group and 12 patients in the LPT group experienced soiling in the long-term post-operative period. The incidence of soiling in the TEPT group (2.1\%) was significantly lower compared with the LPT group $(15.6 \%$; $<<0.05)$. This problem was improved within 6-12 months of functional training and bowel management.

Reports of recurrent long-term constipation in were significantly lower in the LPT group (6.4\%) compared with the TEPT group $(17.0 \%$; $\mathrm{P}<0.05)$. No incontinence was reported for either group. In addition, 9 patients $(9.6 \%)$ in the TEPT group and $14(17.9 \%)$ in the LPT group experienced recurrent enterocolitis in the late post-operative period $(\mathrm{P}=0.108)$. There were 8 cases of grade I enterocolitis $(8.5 \%)$ in the TEPT group and 9 cases $(11.5 \%)$ in the LPT group $(\mathrm{P}=0.610)$; 1 case $(1.1 \%)$ of grade II enterocolitis in the TEPT group and 4 cases $(5.1 \%)$ in the LPT group $(\mathrm{P}=0.178)$; and 1 case $(1.3 \%)$ of grade III enterocolitis in the LPT group and 0 cases in the TEPT group $(\mathrm{P}=0.453)$.

The patients' parents participated in telephone interviews regarding stooling patterns following surgery. A complete long-term outcome questionnaire was obtained for 116 patients with HD, including 64/94 patients in the TEPT group and $52 / 78$ patients in the LPT group. A total of 114 patients $(98.3 \%)$ provided scores in the good-to-excellent range. A total of 90 patients $(77.6 \%)$ had an excellent score, among which 52 patients $(81.3 \%)$ were in the TEPT group and 38 patients $(73.1 \%)$ were in the LPT group; 24 patients $(20.7 \%)$ achieved a good score, with 12 patients $(18.8 \%)$ in the TEPT group and 12 patients $(23.1 \%)$ in the LPT group; 2 patients $(1.7 \%)$ had a fair score, which only occurred in the LPT group $(3.8 \%)$. There was no significant difference between groups in the questionnaire outcome (Table V). Furthermore, no significant differences were observed in post-operative daily bowel movements for 6 months $(\mathrm{P}>0.05)$. However, for the 9 months to 2 years period, significantly more daily bowel movements were reported in the TEPT group compared with the LPT group $(\mathrm{P}<0.05)$. For both procedures, the number of daily 
Table III. Early postoperative complications.

\begin{tabular}{|c|c|c|c|}
\hline Complication & TEPT (n=94) & LPT (n=78) & P-value \\
\hline Perianal dermatitis, n (\%) & $27(25.6)$ & $20(28.7)$ & 0.652 \\
\hline Uroschesis, n (\%) & $4 \quad(4.3)$ & $2(2.6)$ & 0.547 \\
\hline Anastomotic leakage, n (\%) & $0 \quad(0)$ & $0 \quad(0)$ & $<0.001$ \\
\hline Anastomotic strictures, n (\%) & $0 \quad(0)$ & $0 \quad(0)$ & $<0.001$ \\
\hline Early intestinal obstruction, n (\%) & $0 \quad(0)$ & $3(3.8)$ & 0.055 \\
\hline Enterocolitis rate, n (\%) & $19(20.2)$ & $25(32.1)$ & 0.076 \\
\hline \multicolumn{4}{|l|}{ Enterocolitis grade, n (\%) } \\
\hline I & $12(12.8)$ & $14(17.9)$ & 0.345 \\
\hline II & $6 \quad(6.4)$ & $9(11.5)$ & 0.283 \\
\hline III & $1 \quad(1.1)$ & $4(5.1)$ & 0.178 \\
\hline
\end{tabular}

TEPT, transanal endorectal stepwise and gradient cutting muscular cuff pull-through; LPT, laparoscopy-assisted Soave pull-through.

Table IV. Late postoperative complications.

\begin{tabular}{|c|c|c|c|}
\hline & TEPT $(\mathrm{n}=94)$ & LPT $(n=78)$ & P-value \\
\hline Anastomotic strictures, n (\%) & $8(8.5)$ & $7(9.0)$ & 0.915 \\
\hline Incidence of soiling, n (\%) & $2(2.1)$ & $12(15.6)$ & 0.001 \\
\hline Constipation, n (\%) & $16(17.0)$ & $5 \quad(6.4)$ & 0.034 \\
\hline Incontinence in children $>5$ years, $n(\%)$ & $0 \quad(0)$ & $0 \quad(0)$ & $<0.001$ \\
\hline Enterocolitis rate, $\mathrm{n}(\%)$ & $9(9.6)$ & $14(17.9)$ & 0.108 \\
\hline \multicolumn{4}{|l|}{ Enterocolitis grade, n (\%) } \\
\hline I & $8(8.5)$ & $9(11.5)$ & 0.610 \\
\hline II & $1(1.1)$ & $4(5.1)$ & 0.178 \\
\hline III & $0 \quad(0)$ & 1 (1.3) & 0.453 \\
\hline
\end{tabular}

TEPT, transanal endorectal stepwise and gradient cutting muscular cuff pull-through; LPT, laparoscopy-assisted Soave pull-through.
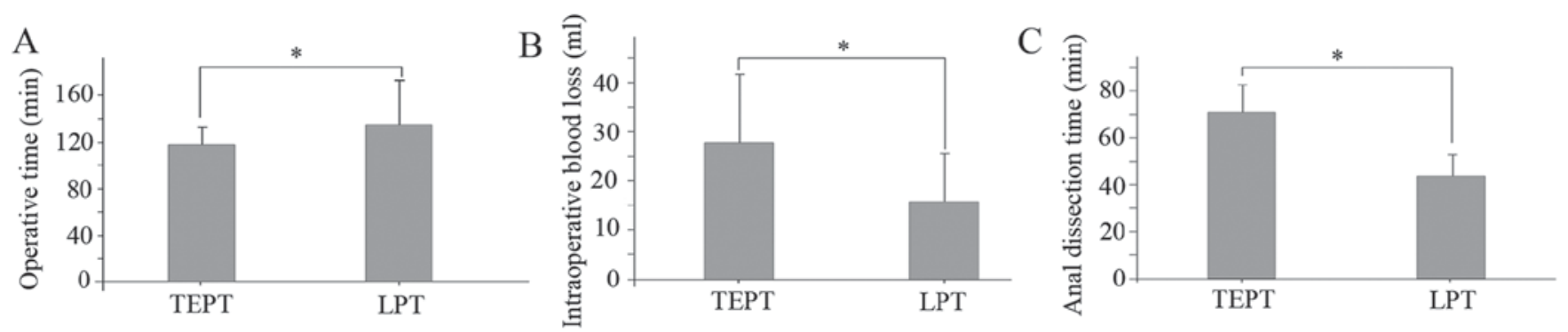

$\mathrm{D}$

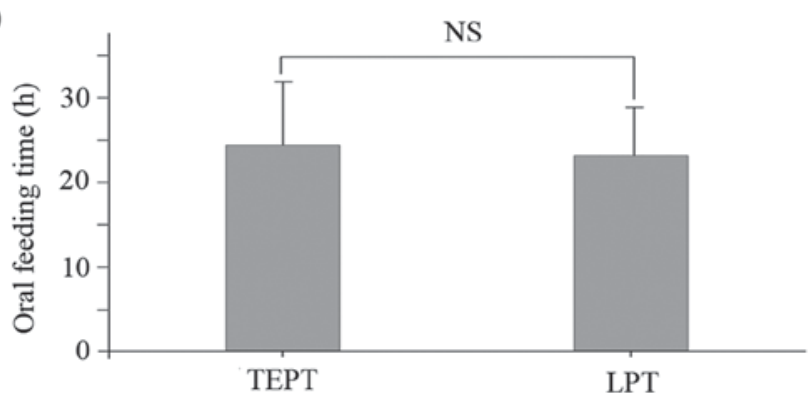

E

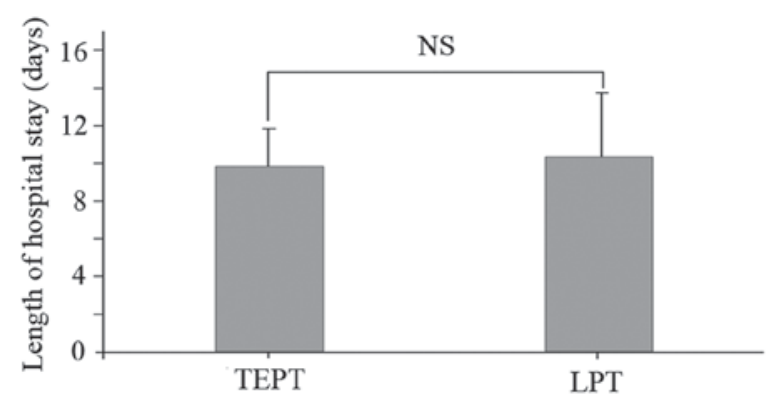

Figure 1. Operative data for the TEPT and LPT groups, including (A) operative time, (B) intraoperative blood loss, (C) anal dissection time, (D) oral feeding time and (E) duration of hospital stay. ${ }^{*} \mathrm{P}<0.05$. TEPT, transanal endorectal stepwise and gradient cutting muscular cuff pull-through; LPT, laparoscopy-assisted Soave pull-through; NS, not significant. 
Table V. Total stooling scores of various groups.

\begin{tabular}{lccc}
\hline & $\mathrm{n}(\%)$ & TEPT, n (\%) & LPT, n (\%) \\
\hline Excellent (0-10) & $90(77.6)$ & $52(81.3)$ & $38(73.1)$ \\
Good (11-20) & $24(20.7)$ & $12(18.8)$ & $12(23.1)$ \\
Fair (21-30) & $2(1.7)$ & $0(0)$ & $2(3.8)$
\end{tabular}

TEPT, transanal endorectal stepwise and gradient cutting muscular cuff pull-through; LPT, laparoscopy-assisted Soave pull-through.

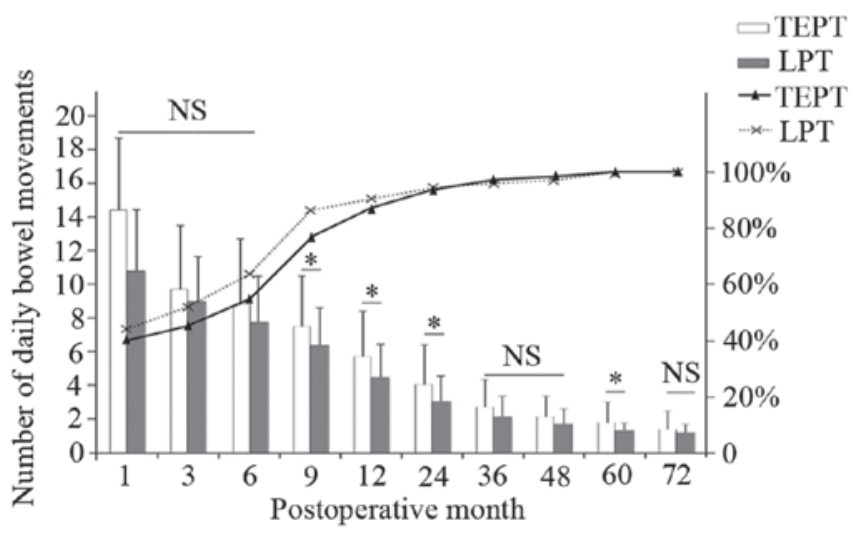

Figure 2. Mean number of daily bowel movements following TEPT or LPT over time. ${ }^{*} \mathrm{P}<0.05$. NS, not significant; TEPT, transanal endorectal stepwise and gradient cutting muscular cuff pull-through; LPT, laparoscopy-assisted Soave pull-through.

bowel movements decreased each year; by 6 years, there was no significant difference between the two groups (Fig. 2).

\section{Discussion}

In recent decades, there have been a number of improvements to surgical methods $(13,19,20)$. This study presents important refinements for stepwise and gradient cutting muscular cuff methods. From September 2003 to August 2014, 172 patients were admitted to the Department of Pediatric Surgery at The Affiliated Hospital of Zunyi Medical College and treated using one of two procedures. Prior to the start of the study, the surgeons involved had gained equal experience in LPT and TEPT, had mastered the various specifications and protocols of these two procedures and had gained the same level of expertise in performing both techniques. Short- and long-term survival outcomes of patients with HD treated with TEPT and LPT were evaluated in this study. To standardize the post-operative long-term evaluation of HD patients, a questionnaire was designed containing 12 questions to assess long-term complications and families of patients who were $\geq 3$ years old were asked to judge bowel habits and continence ability.

TEPT and LPT procedures are becoming increasingly essential techniques for pediatric surgery, the most important advantage of which is the low risk of post-operative scarring (21). However, it remains uncertain which method is the best choice; selections are typically made based on the surgeon's skill and experience. At the Affiliated Hospital of Zunyi Medical College, TEPT was the preferred procedure between 2003 and 2009, whereas LPT is now the favored procedure. The Soave technique has potential issues with the muscular cuff remaining and damage to the anal sphincter, which may lead to constipation, soiling and incontinence (16). As such, the Soave procedure was modified in the present study to the stepwise and gradient cutting muscular cuff method, allowing the retention of $1-2 \mathrm{~cm}$ rectal muscular cuff in neonates and infants, or $3-4 \mathrm{~cm}$ in children with HD. The results revealed that the short-term outcomes were successful and the safety and effectiveness of the transanal procedure has been well documented. In addition, transanal endorectal coloanal anastomosis appears to be simpler and easier to perform with minimal dissection, resulting in less damage to the anal sphincter and pelvic nerves, which is vital for normal defecation (13). Furthermore, opportunities for surgery remain if the transitional zone is at or proximal to the splenic flexure, even in patients with total colonic aganglionosis (6). Starting with laparoscopy is helpful for dividing the right or middle colic vessels, leaving a generous arcade of marginal vessels along the colon and completely dissected colonic ligaments, avoiding over-tension of the pull-though and ischemic colitis (22). The modified Soave method may be applicable for all patients with HD where LPT would have been used before, whereas TEPT can only be adapted to patients for whom the extent of aganglionosis is within the lower rectum or sigmoid colon. The results of the present study suggest that LPT is superior to TEPT in terms of intraoperative blood loss and anal dissection time and that TEPT has a shorter operative time.

In this study, the type and rate of complications were similar in both experimental groups. Although a cited benefit of laparoscopic surgery is the reduction of intestinal obstruction and constipation, three cases of intestinal obstruction were reported; one was colonic volvulus of the pull-through and two were paralytic intestinal obstruction. These occurred in cases of aganglionosis that had extended further than the descending colon and required further ascending colon reversal; the patient with colonic volvulus required further surgery. As previously reported in a 29-year tracked review of long-term results following treatments for HD (23), up to $40 \%$ of patients reported persistent constipation. However, in the present study the rate of constipation in the LPT group was significantly lower compared with the TEPT group (5 vs. 16). The incidence rate recorded in this study was concordant with previous reports, ranging from $6-27 \%$ (24-26). The most common reason for recurrence was a segment of the aganglionic bowel remaining post-surgery (27). LPT is the preferred technique at the Affiliated Hospital of Zunyi Medical College as, apart from the excellent cosmetic results, it allows for biopsies to be obtained immediately and provides adequate post-operative long-term mobilization of the bowel. Therefore, we dissected the pathological colon with stricture and full expansion of the colon, to allow the mobilization of the colon as much as possible. In the TEPT group, 4 patients underwent further surgery as their post-operative biopsy specimens revealed that the reason for constipation was residual aganglionosis of the bowel. All other patients were conservatively treated using comprehensive measures, including diet control, laxatives or anal dilation for $>1$ year.

The incidence of perianal dermatitis and soiling in our cohort was within the range described in other 
publications (28). Rescorla et al (29) suggested that $88 \%$ of patients experienced some difficulties within $<5$ years after a pull-through operation, including temporary soiling and perianal dermatitis. However, the soiling and perianal dermatitis improved in nearly $99 \%$ of patients with a follow-up $>3$ years, which indicates that a better prognosis is possible over time.

One major complication of the traditional transanal Soave technique is the risk of post-operative anastomotic obstruction (30). A procedure modification involving a short muscular cuff anastomosis and dorsal splitting of the muscular wall is advised (31). Simultaneously, performing the heart-shaped anastomosis of the ganglionated proximal end $0.5-1.0 \mathrm{~cm}$ above the dentate line can effectively avoid post-operative anastomotic stricture. In the present study, a stepwise and gradient cutting muscular cuff technique was performed, which left a short cuff remaining. All patients were treated with routine anal dilation for $>6$ months and none required further surgery because of the anastomotic obstruction. If anastomotic obstruction was serious and did not alleviate with routine anal dilation, the patients required additional surgery.

The recently reported incidence rates for enterocolitis range from 4.6-54\% in multiple studies $(30,32)$. Kim et al (30) reported a multicenter study with long-term results, in which the rate of enterocolitis was $22 \%$ with a complete transanal pull-through procedure. In the present study, the rate of enterocolitis was comparable to a previously reported systematic review and meta-analysis that compared a total transanal endorectal pull-through $(22.5 \%)$ with a pull-through with any form of laparoscopic assistance (28.1\%) in infants with HD (10).

Although the incidence of enterocolitis in the present study did not significantly differ between the groups, a slightly higher incidence was observed in the LPT group, which may be partially attributed to the long segments of aganglionosis. The immaturity of the gastrointestinal immune system in aganglionic segments is certainly an important factor in the occurrence and development of enterocolitis (33). Although the association between these covariates and enterocolitis is unknown, it could be postulated that, in patients with anastomotic stricture, obstruction may lead to the development of bacterial overgrowth and enterocolitis. The variable rates of enterocolitis reported may be associated with inconsistent definitions between studies. Conservative treatments for enterocolitis were successful in the majority of patients, whereas further surgical treatment was required in the remaining 3 patients. The present study reveals that the modified technique is a safe and less-invasive procedure than the traditional Soave method, with a low incidence of post-operative enterocolitis compared with previous surgical techniques.

The present study demonstrates that the stepwise and gradient cutting muscular cuff method results in a good functional outcome for extended HD. A transient increase in the frequency of bowel motions during the early post-operative weeks was reported. Bowel movements resulted in average of 7-10 stools/day at 6 months post-surgery, whereas just 5-6 stools were reported in other studies (25). This is probably caused by damage to the anal sphincter during mucosectomy when pulling through the hypertrophic colon. Similar to previous studies, stooling pattern scores and the frequency of bowel movements herein tended to improve with time and may eventually return to a normal range (20). In the present study, it was observed that the number of bowel movements reported in the LPT group was significantly higher compared with the TEPT group from 9 months to 2 years post-surgery. It could be speculated that laparoscopy allows dissection below the peritoneal reflection, which may decrease anal dissection time and avoid injury to the anal sphincter.

In conclusion, the stepwise and gradient cutting muscular cuff method should be considered as an acceptable and safe procedure for the treatment of HD. It was demonstrated that the LPT procedure is feasible for short-segment, classic and extended HD; however, the TEPT procedure is more feasible for short-segment HD. These results demonstrate that LPT is a better surgical approach for children with HD and may be a superior choice to TEPT in the future.

\section{Acknowledgements}

The authors are grateful to all study participants.

\section{Funding}

The present study was funded by the National Natural Science Fund of China (grant no. 81650029).

\section{Availability of data and materials}

The technical appendix and statistical datasets are available from the corresponding author. No additional data are available.

\section{Authors' contributions}

$\mathrm{ZZ}, \mathrm{FZ}$ and $\mathrm{ZJ}$ performed the majority of experiments. MG, YM and YQ analyzed the data. YL conceived and designed the experiments and contributed reagents, methods and analysis tools. All authors authored and reviewed drafts of the paper, and approved the final draft.

\section{Ethics approval and consent to participate}

The Ethics Committee of Zunyi Medical University has reviewed and approved this study. Prior to signing the consent form by the parents, they were fully informed about the surgical procedure, associated risks and possibility for conversion to the open surgical technique.

\section{Patient consent for publication}

All the participants gave informed consent for data sharing when they enrolled in this study.

\section{Competing interests}

The authors report no relevant competing interest.

\section{Authors' information}

Dr. Zebing Zheng, Department of Pediatric General Thoracic and Urinary Surgery, Affiliated Hospital of Zunyi Medical 
College. 149 Dalian Road, Zunyi, Guizhou 563000, P.R. China. E-mail:Zebing1988@sina.com. Telephone: +86-851-28608446.

\section{References}

1. Stensrud KJ, Emblem R and Bjonland K: Anal endosonography and bowel function in patients undergoing different types of endorectal pull-through procedures for Hirschsprung disease. J Pediatr Surg 50: 1341-1346, 2015.

2. Zhang S, Li J, Wu Y, Hu Y, Duan C, Wang M and Gai Z: Comparison of laparoscopic-assisted operations and laparotomy operations for the treatment of hirschsprung disease: evidence from a meta-analysis. Medicine (Baltimore) 94: e1632, 2015.

3. Tomuschat C, Zimmer J and Puri P: Laparoscopic-assisted pull-through operation for Hirschsprung's disease: A systematic review and meta-analysis. Pediatr Surg Int 32: 751-757, 2016.

4. Xiong X, Chen X, Wang G and Feng J: Long term quality of life in patients with Hirschsprung's disease who underwent heart-shaped anastomosis during childhood: A twenty-year follow-up in China. J Pediatr Surg 50: 2044-2047, 2015.

5. Puri P: Hirschsprung's disease. In: Pediatric Surgery. Puri $P$ and Höllwarth M (eds). Springer, Berlin, pp275-288, 2005.

6. Zhang JS, Li L, Hou WY, Liu SL, Diao M, Zhang J, Ming AX and Cheng W: Transanal rectal mucosectomy and partial internal anal sphincterectomy for Hirschsprung's disease. J Pediatr Surg 49: 831-834, 2014.

7. Langer JC, Minkes RK, Mazziotti MV, Skinner MA and Winthrop AL: Transanal one-stage Soave procedure for infants with Hirschsprung's disease. J Pediatr surg 34: 148-151, 1999.

8. De La Torre L and Ortega A: Transanal versus open endorectal pull-through for Hirschsprung's disease. J Pediatr surg 35: $1630-1632,2000$.

9. Georgeson KE, Cohen RD, Hebra A, Jona JZ, Powell DM, Rothenberg SS and Tagge EP: Primary laparoscopic-assisted endorectal colon pull-through for Hirschsprung's disease: A new gold standard. Ann Surg 229: 678-683, 1999.

10. Thomson D, Allin B, Long AM, Bradnock T, Walker G and Knight M: Laparoscopic assistance for primary transanal pull-through in Hirschsprung's disease: A systematic review and meta-analysis. BMJ Open 5: e006063, 2015.

11. Granström AL, Husberg B, Nordenskjöld A, Svensson PJ and Wester T: Laparoscopic-assisted pull-through for Hirschsprung's disease, a prospective repeated evaluation of functional outcome. J Pediatr surg 48: 2536-2539, 2013.

12. Khazdouz M, Sezavar M, Imani B, Akhavan H, Babapour A and Khademi G: Clinical outcome and bowel function after surgical treatment in Hirschsprung's disease. Afr J Paediatr Surg 12: 143-147, 2015.

13. Zhang SC, Bai YZ, Wang W and Wang WL: Clinical outcome in children after transanal 1-stage endorectall pull-through operation for Hirschsprung's disease. J Pediatr Surg 40: 1307-1311, 2005.

14. Elhalaby EA, Teitelbaum DH, Coran AG and Heidelberger KP: Enterocolitis associtated with Hirschsprung's disease: A clinical histopathological correlative study. J Pediatr Surg 30: 1023-1027, 1995.

15. El-Sawaf MI, Drongowski RA, Chamberlain JN, Coran AG and Teitelbaum DH: Are the long-term results of the transanal pull-through equal to those of the transabdominal pull-through? A comparison of the 2 approaches for Hirschsprung's disease. J Pediatr Surg 42: 41-47, 2007.

16. Dickie BH, Webb KM, Eradi B and Levitt MA: The problematic Soave cuff in Hirschsprung disease: Manifestations and treatment. J Pediatr Surg 49: 77-80, 2014.

17. Rouzrokh M, Jadali F, Gharib A, Khaleghnejad-Tabari A, Tavassoli A and Mohajerzadeh L: Can we rely on frozen sections of a rectal biopsy for one-stage trans-anal pull-through operation in hirschsprung's disease? Iran J Pediatr 21: 72-76, 2011.
18. Agrawal RK, Kakkar N, Vasishta RK, Kumari V, Samujh R and Rao KL: Acetylcholinesterase histochemistry (AChE)-A helpful technique in the diagnosis and in aiding the operative procedures of Hirschsprung disease. Diagn Pathol 10: 208, 2015.

19. Podevin G, Lardy H, Azzis O, Branchereau S, Petit T, Sfeir R, Weil D, Heloury Y and Fremond B: Technical problems and complications of transanal pull-through for Hirschsprung's disease. Eur J Pediatr Surg 16: 104-108, 2006.

20. Ishihara M, Yamataka A, Kaneyama K, Koga H, Kobayashi H, Lane GJ and Miyano T: Prospective analysis of primary modified Georgeson's laparoscopy-assisted endorectal pull-through for Hirschsprung's disease: Short-to mid-term results. Pediatr Surg Int 21: 878-882, 2005.

21. Tang ST, Wang GB, Cao GQ, Wang Y, Mao YZ, Li SW, Li S, Yang Y, Yang J and Yang L: 10 years of experience with laparoscopic-assisted endorectal Soave pull-through procedure for Hirschsprung's disease in China. J Laparoendosc Adv Surg Tech A 22: 280-284, 2012.

22. Aubdoollah TH, Li K, Zhang X, Li S, Yang L, Lei HY, Dolo PR, Xiang XC, Cao GQ, Wang GB and Tang ST: Clinical outcomes and ergonomics analysis of three laparoscopic techniques for Hirschsprung's disease. World J Gastroenterol 21: 8903-8911, 2015.

23. Menezes M, Corbally M and Puri P: Long-term results of bowel function after treatment for Hirschsprung's disease: A 29-year review. Pediatr Surg Int 22: 987-990, 2006.

24. Huang Y, Zheng S and Xiao X: A follow-up study on postoperative function after a transanal Soave 1-stage endorectal pull-through procedure for Hirschsprung's disease. J Pediatr Surg 43: 1691-1695, 2008.

25. Ishikawa N, Kubota A, Kawahara H, Hasegawa T, Okuyama H, Uehara S and Mitani Y: Transanal mucosectomy for endorectal pull-through in Hirschsprung's disease: Comparison of abdominal, extraanal and transanal approaches. Pediatr Surg Int 24: 1127-1129, 2008

26. Dahal GR, Wang JX and Guo LH: Long-term outcome of children after single-stage transanal endorectal pull-through for Hirschsprung's disease. World J Pediatr 7: 65-69, 2011.

27. Van de Ven TJ, Sloots CE, Wijnen MH, Rassouli R, van Rooij I, Wijnen RM and de Blaauw I: Transanal endorectal pull-through for classic segment Hirschsprung's disease: With or without laparoscopic mobilization of the rectosigmoid? J Pediatr Surg 48: 1914-1918, 2013.

28. Chen Y, Nah SA, Laksmi NK, Ong CC, Chua JH, Jacobsen A and Low Y: Transanal endorectal pullthrough versus transabdominal approach for Hirschsprung's disease: A systematic review and meta-analysis. J Pediatr Surg 48: 642-651, 2013.

29. Rescorla FJ, Morrison AM, Engles D, West KW and Grosfeld JL: Hirschsprung's disease. Evaluation of mortality and long-term function in 260 cases. Arch Surg 127: 934-942, 1992.

30. Kim AC, Langer JC, Pastor AC,Zhang L, Sloots CE, Hamilton NA, Neal MD, Craig BT, Tkach EK, Hackam DJ, et al: Endorectal pull-through for Hirschsprung's disease-a multicenter, long-term comparison of results: Transanal vs transabdominal approach. J Pediatr Surg 45: 1213-1220, 2010.

31. Miyano G, Koga H, Okawada M, Doi T, Sueyoshi R, Nakamura H, Seo S, Ochi T, Yamada S, Imaizumi T, et al: Rectal mucosal dissection commencing directly on the anorectal line versus commencing above the dentate line in laparoscopy-assited transanal pull-through for Hirschsprung's disease: prospective medium-term follow-up. J Pediatr Surg 50: 2041-2043, 2015.

32. Giuliani S, Betalli P, Narciso A,GrandiF, Midrio P, Mognato G and Gamba P: Outcome cpmparison among laparoscopic Duhamel, laparotomic Duhamel and transanal endorectal pull-through: A single-center, 18-year experience. J Laparoendosc Adv Surg Tech A 21: 859-863, 2011.

33. Yan Z, Poroyko V, Gu S, Zhang Z, Pan L, Wang J, Bao N and Hong L: Characterization of the intestinal microbiome of Hirschsprung's disease with and without enterocolitis. Biochem Biophys Res Commun 445: 269-274, 2014. 